\title{
Awareness of smoking in adolescents with inflammatory bowel disease
}

\author{
Marcin Dziekiewicz ${ }^{1, A-F}{ }^{\oplus}$, Kinga Kowalska-Duplaga ${ }^{2, A-F} \oplus$,

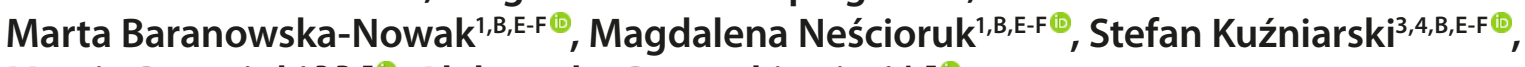 \\ Marcin Banasiuk ${ }^{1, \mathrm{~B}, \mathrm{D}-\mathrm{F} \oplus}$, Aleksandra Banaszkiewicz ${ }^{1, \mathrm{~A}-\mathrm{F} \oplus}$ \\ ${ }^{1}$ Department of Paediatric Gastroenterology and Nutrition, Medical University, Warsaw, Poland \\ ${ }^{2}$ Department of Pediatrics, Gastroenterology and Nutrition, Collegium Medicum, Jagiellonian University, Kraków, Poland \\ ${ }^{3}$ Department of Paediatric Neurosurgery and Traumatology, Medical University, Warsaw, Poland \\ ${ }^{4}$ Student Research Association, Department of Paediatric Gastroenterology and Nutrition, Medical University, Warsaw, \\ Poland \\ A - Research concept and design, B - Collection and/or assembly of data, C - Data analysis and interpretation, \\ $D$ - Writing the article, E - Critical revision of the article, F- Final approval of article
}

\begin{abstract}
Dziekiewicz M, Kowalska-Duplaga K, Baranowska-Nowak M, Neścioruk M, Kuźniarski S, Banasiuk M, Banaszkiewicz A. Awareness of smoking in adolescents with inflammatory bowel disease. Ann Agric Environ Med. 2020; 27(1): 61-65. doi: 10.26444/aaem/105821
\end{abstract}

\begin{abstract}
Introduction and objectives. Inflammatory bowel disease (IBD) is long life disease that results from an interaction between a polygenetic predisposition and environmental factors, including smoking. The aim of this study was to evaluate beliefs about and awareness of smoking among adolescents with IBD compared to healthy controls.

Materials and method. Patients with IBD, Crohn's disease (CD) and ulcerative colitis (UC), and healthy controls were asked to complete a questionnaire on demographic data and smoking status. The questionnaire also included data on beliefs and awareness of smoking-related health effects, including effects on IBD.

Results. A total of 139 IBD patients and 108 controls were enrolled in the study. Of the IBD patients, 17/139 (12.2\%) were smokers compared to $18 / 108(16.7 \%)$ of controls $(p=0.3)$. Patients with IBD were nine times more likely to be everyday smokers than occasional smokers (OR=9.2, 95\% Cl: 1.9- 45.1, $\mathrm{p}=0.004)$. No difference was found between patients with CD and UC in their answers to the question of whether "smoking increases the risk for surgery in your type of IBD" (17/28 (60.7\%) vs. $10 / 29(34.5 \%)$, respectively ( $p=0.047)$. More patients with CD than UC were aware of the risks of smoking on their disease: extra-intestinal manifestations and disease exacerbation, $\mathrm{OR}=11.3(95 \% \mathrm{Cl}: 4.1-30.9 ; \mathrm{p}=0.000)$ and $\mathrm{OR}=19.3(95 \% \mathrm{Cl}$ : 6.7 55.1; $\mathrm{p}=0.000)$, respectively.

Conclusions. The data obtained demonstrated that adolescents with CD are much more aware of the role of smoking on CD than are their peers with UC; however, this awareness is still unsatisfactory. Therefore, there is an unmet need to implement better anti-smoking strategies for this group of patients.
\end{abstract}

\section{Key words}

Crohn's disease, ulcerative colitis, environmental factors, tobacco

\section{INTRODUCTION}

The etiology of inflammatory bowel disease (IBD), including ulcerative colitis (UC) and Crohn's disease (CD), has not been fully described. However, some of the genetic, immunologic and environmental factors that contribute to the development of IBD are well established [1]. Among environmental factors, the role of smoking is one of the most curious. It has already been proven that tobacco smoking has a dichotomous effect on the course of IBD: it is protective for patients with UC and negative for patients with CD $[1,2,3]$. Cigarette smoking significantly reduces risk of UC occurrence, compared to nonsmokers (OR 0.58; 0.45-0.75) [4], diminishes hospitalization, relapse rates, and needs for corticosteroids and colectomy [5]. As for $\mathrm{CD}$, smoking significantly increases the risk of illness prevalence, worsens the course of disease and quality of life. Smokers with CD are more likely to present with extra-intestinal symptoms, to have disease exacerbation and more frequently

Address for correspondence: Kinga Kowalska-Duplaga, Uniwersytet Jagielloński Collegium Medicum, Department of Pediatrics, Gastroenterology and Nut, 30-663, Kraków, Poland

E-mail: kinga.kowalska-duplaga@uj.edu.pl

Received: 13.12.2018; accepted: 18.03.2019; first published:08.04.2019 require hospitalization. They are less responsive to treatment and more prone to developing complications. They also are at higher risk of surgery and of postoperative relapse $[6,7]$.

Importantly, individuals who decide to give up smoking have a similar prognosis as CD patients who never smoked [5]. On the other hand, ex-smokers have increased risk of developing UC [8].

The reasons for these opposing effects of smoking on UC and CD have not been established; however, there are recent data linking the effect of tobacco smoking with genetics [9]. Helbig et al. found that smoking may modulate the functional consequences of the CD-associated polymorphism in NOD2 [10]. Another proposed mechanism of action is associated with a difference in CD and UC immune response (secretion of pro-inflammatory cytokine associated with Th-1 versus Th-2, respectively), differences in microbiome composition, gut permeability and mucosal barrier function $[11,12,13]$. The effects of smoking on the intestinal microbiome have been demonstrated in several studies. There is also growing evidence that smoking may alter non-intestinal microbiome and decrease its diversity. These findings may explain the potential role of smoking in the pathogenesis of extraintestinal IBD symptoms [14]. 
Smoking is a major issue because almost one-third of the adult population in Europe smokes, and only approximately $10 \%$ of them start smoking after 20 years of age. [15] In Poland, $20.6 \%$ of adolescents were found to be active smokers [16]. Data on smoking in patients with IBD are limited and are based on adult data only. These data indicate that awareness of the negative impact of smoking is inadequate among patients with IBD. There is no evidence that smoking has deleterious effects on adolescents with CD; however, it is very probable. Regardless, adolescents with CD become adults with CD, and then this effect is proven. In contrast, adolescents, as opposed to adults, are more likely to smoke for a short period of time and not (yet) in a habitual manner. Therefore, it can be said that quitting smoking, while not easy in all age groups, is relatively easier in adolescents than in adults. It would be worthwhile to prepare programmes of smoking awareness early enough for children and adolescents with IBD to prevent smoking for non-smokers, and encourage quitting for smokers. Such a programme would also be worthwhile for adolescents with UC, because they can believe that smoking is beneficial for them, forgetting that cigarette smoking harms nearly every organ of the body, causes many diseases, including pulmonary and cardiovascular diseases, as well as various types of cancers, and affects the general health of smokers [17].

\section{OBJECTIVES}

We do not know what adolescents with IBD know about smoking and about the impact of smoking on their disease. For these reasons, this study aimed to evaluate the knowledge of adolescent patients with IBD regarding the effects of smoking on IBD.

\section{MATERIALS AND METHOD}

This prospective, controlled study was conducted in two university-affiliated hospitals for children in Warsaw and Kraków, Poland, between April 2016 - June 2017.

The study group was comprised of children and adolescents older than 11 years of age, and diagnosed with IBD at least one year before inclusion into the study. The diagnosis of $\mathrm{CD}$ or UC was based on clinical signs and symptoms, as well as on endoscopic, histological and radiological results, according to the revised Porto criteria [18]. The patients were at different stages of the disease and treatment process. The control group consisted of healthy children of the same age. All study participants were asked to fill in an anonymous one-page questionnaire which consisted of 20 questions. In the first part, three general questions were asked regarding age, gender and type of respondent: healthy control or patient suffering from IBD (with CD or UC). Next two questions were addressed only to the latter group and regarded disease duration and current status of the illness: remission or disease flare.

The study participants were then asked about their smoking behavior: current, former, never or passive smoker. They were also asked if they are occasional or everyday smokers. In the second part of the questionnaire, all study participants were asked their opinion about a list of the potential effects of smoking on health issues, including general health, risk of heart infarct, stroke, influenza, lung cancer, and asthma. Additionally, children and adolescents with IBD were asked if smoking increases the risk for developing IBD, extraintestinal symptoms of IBD and the IBD course, and surgery. Answers included "yes", "no" and "do not know" options.

Continuous variables were expressed as medians (IQRs - interquartile rates) based on the data distribution. The Mann-Whitney U test was used to compare median values between groups. The $\chi 2$ or Fisher's exact test was used to compare proportions. The Yates correction for continuity was used when appropriate. Odds ratio (OR) and 95\% confidence interval (CI) were calculated. Data analysis was conducted using Statistica 12 (Statsoft, Oklahoma, USA).

The study protocol was approved by the Ethics Committee on Research at the Medical University in Warsaw. All patients were informed about the study objectives.

\section{RESULTS}

In total, 139 patients with IBD and 108 controls were enrolled in the study. None of the patients refused to participate, and all of them correctly completed the survey. The clinical characteristics of patients and controls are shown in Table 1. At the time of enrollment, there were no differences in age, gender, disease duration and disease activity between patients with CD and UC. There was also no difference in smoking

Table 1. Clinical characteristics of subjects

\begin{tabular}{|c|c|c|c|c|c|c|}
\hline & IBD & $C D$ & UC & $\begin{array}{l}\text { p-value } \\
\text { CD vs UC }\end{array}$ & Controls & $\begin{array}{c}p \text {-value } \\
\text { IBD vs Controls }\end{array}$ \\
\hline $\mathrm{N}$ & 139 & 84 & 55 & & 108 & \\
\hline Gender (males) & 77 (55.4\%) & $46(54.8 \%)$ & $31(56.4 \%)$ & 0.3 & $49(45.4 \%)$ & 0.1 \\
\hline Disease duration (median years, IQR) & $2(1-6)$ & $2(1-6)$ & $2(1-5)$ & 0.9 & & \\
\hline Patients in remission & $69(49.6 \%)$ & $46(54.8 \%)$ & $23(41.8 \%)$ & 0.1 & & \\
\hline Active smokers & $17(12.2 \%)$ & $7(8.3 \%)$ & $10(18.2 \%)$ & 0.08 & $18(16.7 \%)$ & 0.3 \\
\hline Occasional smokers & $6(35.3 \%)$ & $3(42.9 \%)$ & $3(30 \%)$ & \multirow{2}{*}{0.6} & $15(83.3 \%)$ & \multirow{2}{*}{0.004} \\
\hline Every day smokers & $11(64.7 \%)$ & $4(57.1 \%)$ & $7(70 \%)$ & & $3(16.7 \%)$ & \\
\hline Quit smoking Attempts & $4(23.5 \%)$ & $2(28.6 \%)$ & $2(20 \%)$ & 1.0 & $13(72.2 \%)$ & 0.005 \\
\hline Passive smokers & 45 (32.4\%) & 30 (35.7\%) & $15(27.3)$ & 0.3 & 35 (32.4\%) & 1.0 \\
\hline
\end{tabular}

IBD - inflammatory bowel disease; CD - Crohn's disease; UC - ulcerative colitis, IQR interquartile rate 
behaviour between patients with CD and UC. However, there were more occasional smokers and attempts to quit smoking in the control group than in the IBD group $(\mathrm{p}=0.004$ and $\mathrm{p}=0.005$, respectively). Patients with IBD were nine times more likely to be everyday smokers than occasional smokers $(\mathrm{OR}=9.2$, 95\% CI: 1.9 - 45.1; $\mathrm{p}=0.004)$. Taking patients with IBD and controls together, smokers were older compared to non-smokers (median age 17 years (IQR: 16-17) vs. 15 years (IQR: $14-16 ; \mathrm{p}=0.000)$ and were twice as likely to be males $(\mathrm{OR}=2.6,95 \%$ CI: $1.1-5.1 ; \mathrm{p}=0.03)$.

Figure 1 shows the results of replies to questions directed to all study participants. There were no differences in answers to questions about the impact of smoking on health issues, including general health, risk of myocardial infarction, influenza, lung cancer or asthma, among patients with CD, patients with UC and controls. More controls determined smoking as a risk factor for stroke, compared to patients with IBD (93.7\% vs. $79.2 \%$, respectively; $\mathrm{p}=0.02)$; there was no difference between CD and UC patients $(\mathrm{p}=0.9)$.

In the questions below, for the statistical assessment, we excluded only "do not know" answers. Patients' knowledge regarding the effects of smoking on IBD is presented in Figure 2. No differences were found between CD and UC patients in correct answers to questions regarding whether smoking increases the risk for developing CD: $17 / 49$ (34.7\%) vs. $8 / 24(33.3 \%)$, respectively $(\mathrm{p}=0.09)$. Both $\mathrm{CD}$ and UC patients were aware that smoking decreases the risk for developing UC: $32 / 46$ (69.6\%) vs. $21 / 28$ (75\%), respectively $(\mathrm{p}=0.6)$.

No differences were also between CD and UC patients in answers to the question whether "smoking increases the risk for surgery in your type of IBD” (17/28 (60.7\%) vs. 10/29 (34.5\%), respectively $(\mathrm{p}=0.47)$.

To the questions: "Do you think that smoking increases the risk for extra-intestinal symptoms in your type of IBD?" and "Do you think that smoking may cause a more severe course of your type of IBD?", patients with CD were 11 times and 19 times more likely to give the correct answers, compared to patients with UC: $\mathrm{OR}=11.29$ (95\%CI: $4.12-30.89$; $\mathrm{p}=0.000)$ and $\mathrm{OR}=19.33$ (95\% CI: $6.71-55.09 ; \mathrm{p}=0.000)$, respectively.

There were no differences between smokers and nonsmokers regarding responses to all questions, with one exception: non-smokers more frequently gave the correct answer to question about the impact of smoking on asthma

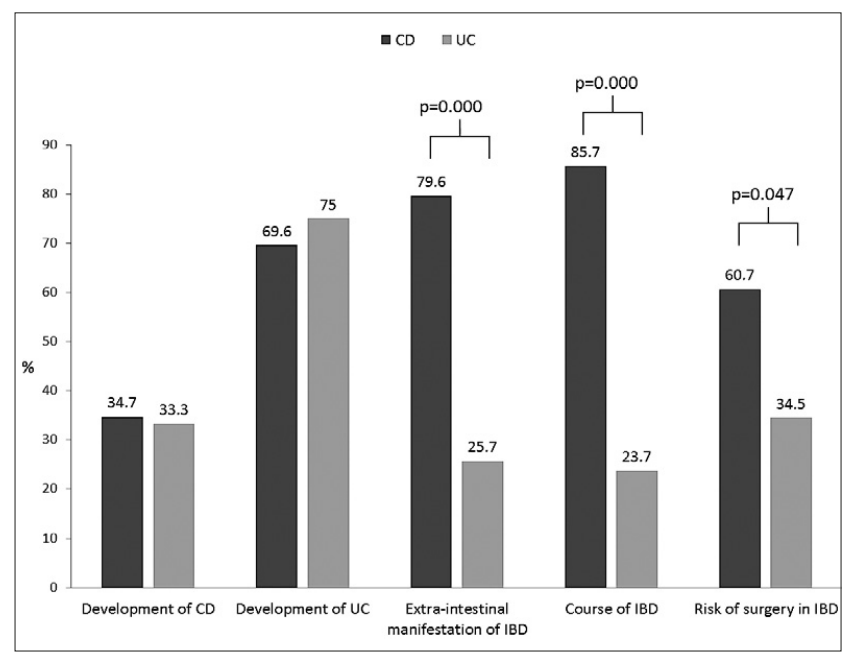

Figure 2. Patient's beliefs about the effect of smoking on inflammatory bowel disease (expressed as \% of correct answers: "yes" for patients with CD and "no" for patients with UC)

CD - Crohn's disease; UC - ulcerative colitis; IBD - inflammatory bowel disease

( $97 \%$ vs. $82.1 \%$; $\mathrm{p}=0.005$ ). $73.3 \%$ of passive smokers, but none of active smokers, knew that smoking increases the risk of extra-intestinal manifestation $(\mathrm{p}=0.01)$.

\section{DISCUSSION}

The results of this prospective trial demonstrate that knowledge regarding the deleterious effects of smoking on general health is good among adolescents with IBD; however, it is strongly insufficient with regard to the impact of smoking on their disease. To-date, a few studies assessing the awareness of smoking in adult patients with IBD have already been published [19, 20, 21, 22].

In this study, more than $95 \%$ of adolescents with IBD, both $\mathrm{CD}$ and UC, knew that smoking affects general health and asthma control, and increases the risk of myocardial infarction and lung cancer. Fewer IBD adolescents (although still almost $80 \%$ ) knew that smoking increases the risk of stroke. Less than half of the respondents (41.1\%) were aware of the fact that smoking increases the risk of influenza. The obtained results are in line with the results of studies in

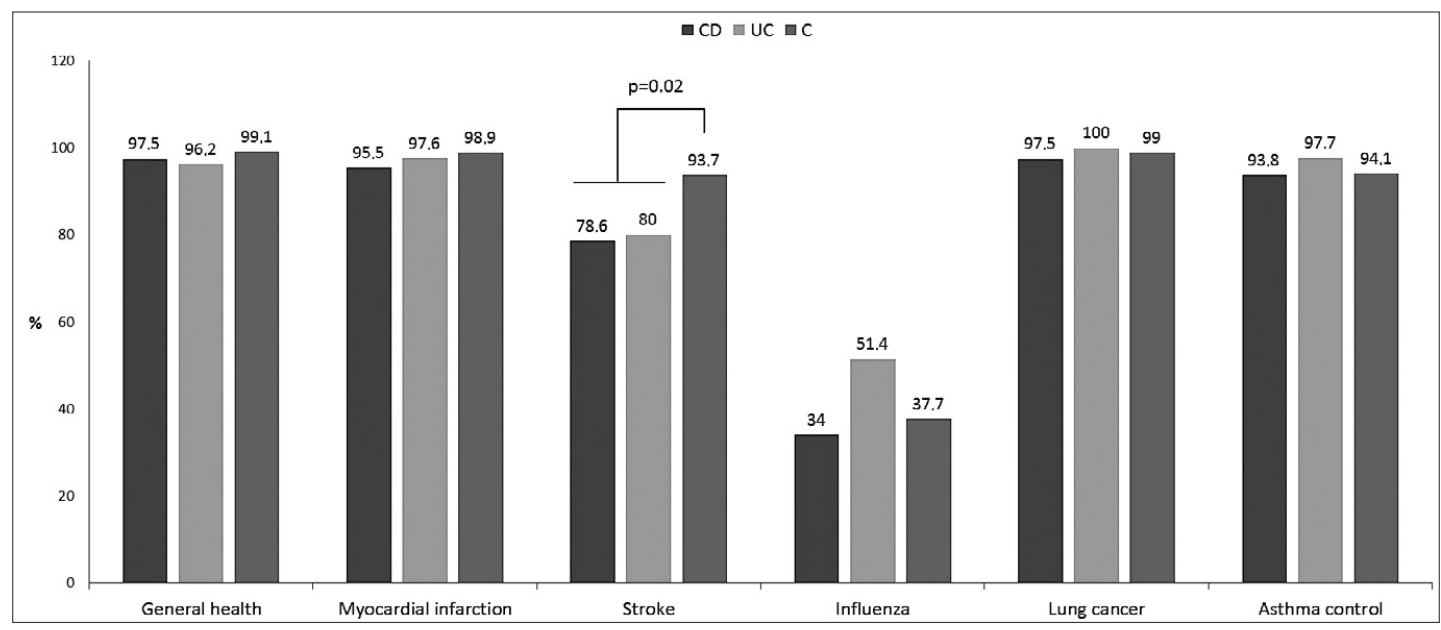

Figure 1. Patient's awareness about smoking as a risk factor for general health issues (expressed as \% of "yes" answers).

CD - Crohn's disease; UC - ulcerative colitis; C - control 
adults with IBD; between $89 \%$ - $98 \%$ of adult patients thought that smoking increases the risk of lung cancer and cardiovascular disease $[20,22]$. Moreover, in the only adult study that involved questions about smoking and influenza, the results were very similar to those in the current study (32\% of adult IBD patients vs. $41.7 \%$ in the current study) [20].

Similar to this study, there was also no difference between the answers of IBD patients and those of controls. The only significant difference found in the results of this part of the questionnaire was the fact that more controls found smoking to be a risk factor for stroke, compared to patients with IBD; however, the rate was high in both groups and there was no difference between CD and UC patients. de Bie et al. found a very similar association: $87 \%$ of controls recognized smoking as a risk factor for stroke, compared to $81 \%$ of patients with CD [20]. Patients with IBD, similar to the general population, were strongly aware of the adverse effects of smoking on general health [23]. In the presented study, approximately one-third of patients with IBD gave correct answers to the question of whether smoking increases the risk of developing $\mathrm{CD}$. Twice as many patients believed that smoking does not influence the onset of UC. The results of studies conducted in adults are differential. Between 9.5\% [22] and 64.3\% [21] of patients with IBD gave the correct answers regarding this issue. In the studies in which patients with CD were aware of the role of smoking in promoting the development of their disease, patients with UC were not aware of this [19, 20,24]. When patients with CD were not aware of the influence of smoking on the onset of their disease, the majority of patients with UC knew about the preventive role of smoking in the development of UC [21]. The rates of awareness about smoking effect on the onset of IBD is still unsatisfactory. In the opinion of the authors of the current study, it is much more difficult to explain to patients with UC that they should not smoke, although tobacco use my have a protective effect on their disease.

This study shows that many more adolescents with CD knew that smoking affects their disease than did their peers with UC. Similar findings were reported by DucharmeBenard et al. in their Canadian cohorts of adults with IBD [19]. In contrast, only a minority of British and French patients with CD recognized that smoking increases the risk of additional operations for their disease [21,22]. The most plausible explanation is the different level of knowledge about smoking between adolescents with CD and UC. However, the authors of the current study concede that they suspect that the proper answers given by adolescents with IBD are not the result of higher disease-related knowledge, but rather the common opinion that "smoking is bad" and is therefore also "bad for CD and bad for UC". However, the authors have no proof for their suspicion. Moreover, although the study group comprised of more frequent UC smokers than CD smokers, no differences were found in knowledge of the impact of smoking on the course of IBD between smokers and non-smokers. This finding indicates that knowledge about smoking as an exacerbating factor of IBD concerns patients with both types of the disease equally.

In this study, $12 \%$ of patients declared that they were current smokers. It is believed that these data are accurate because the questionnaire was anonymous, and parents were requested to allow their children to complete the questionnaire in isolation. Similar rates of smoking (16\%) were also found in controls. This finding indicates that smoking is less popular in adolescents than in adults. Saadoune et al. reported that in their groups of patients with IBD, $31 \%$ were smokers [21], and Ryan et al. reported that 32\% were smokers [22]. In Poland in 2014,almost one-third of the population were to be active smokers, and the majority of them started to smoke in adolescence and young adulthood [25]. Attempts to quit smoking were statistically more frequent in the control group than in the IBD group. This be a result of the fact that there were many more occasional smokers in the control group than in the patients' group. A lower number of cigarettes smoked per day is a known predictor of smoking cessation [26].

The results of this study emphasize the need for health professionals to organize systematic education for patients with IBD regarding the risk of smoking associated with their disease. Moreover, smoking cessation programmes should be routinely incorporated into the management of IBD from the time of IBD diagnosis.

The main advantage of this study is the fact that, for the first time, knowledge of the adverse effects of smoking on IBD among a group of adolescents with IBD has been assessed. The study groups were representative because all patients and controls, and their parents who were invited to participate in the study, agreed to do so.

However, the study also has shortcomings. The study participants were not asked about the sources of their knowledge on smoking. Therefore, it was not possible to determine whether doctors or nurses gave any support to adolescents' decisions to smoke or not. Also, were not asked if their decision to quit smoking was associated with the advice of doctors or nurses. Moreover, it was not possible to explain why attempts to quit smoking were more frequent in the control group than in the IBD group $(p=0.005)$. Unfortunately, on the basis of this study, the role of the medical staff in the participants' knowledge about the harmful effects of smoking could not be assessed.

In conclusion, the data obtained demonstrate that adolescents with CD and UC are aware of the harmful effects of smoking on general health, influenza, asthma, myocardial infarction and lung cancer. Adolescents with $\mathrm{CD}$ are much more aware of the role of smoking on $\mathrm{CD}$, compared to their peers with UC; however, this awareness is still unsatisfactory. There is an unmet need to implement routine educational strategies for this group of patients.

\section{REFERENCES}

1. Shouval DS, Rufo PA. The Role of environmental factors in the pathogenesis of inflammatory bowel diseases. JAMA Pediatr. 2017; 171: 999.

2. Parkes GC, Whelan K, Lindsay JO. Smoking in inflammatory bowel disease: Impact on disease course and insights into the aetiology of its effect. J Crohn's Colitis. 2014; 8: 717-725.

3. van der Slot K, Amini M, Peters V, Dijkstra G, Alizadeh BZ. Inflammatory bowel diseases: review of known environmental protective and risk factors involved. Inflamm Bowel Dis. 2017; 9: 1499-1509.

4. Mahid SS, Minor KS, Soto RE, Hornung CA, Galandiuk S. Smoking and inflammatory bowel disease: a meta-analysis. Mayo Clin Proc. 2006; 81: 1462-71.

5. Cosnes J, Beaugerie L, Carbonnel F, Gendre J. Smoking cessation and the course of Crohn's disease: An intervention study. Gastroenterology. 2001; 120: 1093-9.

6. Reese GE, Nanidis T, Borysiewicz C, Yamamoto T, Orchard T, Tekkis PP. The effect of smoking after surgery for Crohn's disease: a metaanalysis of observational studies. Int J Colorectal Dis. 2008; 23: 1213-21. 
7. Cosnes, Carbonnel, Carrat, Beaugerie, Cattan, Gendre. Effects of current and former cigarette smoking on the clinical course of Crohn's disease. Aliment Pharmacol Ther. 1999; 13: 1403-11.

8. Persson PG, Ahlbom A, Hellers G. Inflammatory bowel disease and tobacco smoke--a case-control study. Gut. 1990; 31: 1377-81.

9. Yadav P, Ellinghaus D, Rémy G, Freitag-Wolf S, Cesaro A, Degenhardt $\mathrm{F}$, et al. Genetic factors interact with tobacco smoke to modify risk for inflammatory bowel disease in humans and mice. Gastroenterology. 2017; 153: 550-65.

10. Helbig KL, Nothnagel M, Hampe J, Balschun T, Nikolaus S, Schreiber $\mathrm{S}$, et al. A case-only study of gene-environment interaction between genetic susceptibility variants in NOD2 and cigarette smoking in Crohn's disease aetiology. BMC Med Genet. 2012; 13: 14.

11. Benjamin JL, Hedin CRH, Koutsoumpas A, Ng SC, McCarthy NE Prescott NJ, et al. Smokers with active Crohn's disease have a clinically relevant dysbiosis of the gastrointestinal microbiota. Inflamm Bowel Dis. 2012; 18: 1092-100.

12. Rom O, Avezov K, Aizenbud D, Reznick AZ. Cigarette smoking and inflammation revisited. Respir Physiol Neurobiol. 2013; 187: 5-10.

13. Berkowitz L, Schultz BM, Salazar GA, Pardo-Roa C, Sebastián VP, Álvarez-Lobos MM, et al. Impact of cigarette smoking on the gastrointestinal tract inflammation: Opposing effects in Crohn's disease and ulcerative colitis. Front. Immunol. 2018; 9: 74.

14. Savin Z, Kivity S, Yonath H, Yehuda S. Smoking and the intestinal microbiome. Arch Microbiol. 2018; 200: 677-84.

15. Gallus S, Lugo A, La Vecchia C, Boffetta P, Chaloupka FJ, Colombo $\mathrm{P}$, et al. Pricing policies and control of tobacco in Europe (PPACTE) project. Eur J Cancer Prev. 2014; 23: 177-85.

16. Nowak M, Papiernik M, Mikulska A, Czarkowska-Paczek B. Smoking, alcohol consumption, and illicit substances use among adolescents in Poland. Subst Abuse Treat Prev Policy. 2018; 13: 42.
17. General US. U.S. Department of Health and Human Services. How tobacco smoke causes disease: the biology and behavioral basis for smoking-attributable disease. A report of the surgeon general. Public Heal Serv Off Surg Gen 2010.

18. Levine A, Koletzko S, Turner D, Escher JC, Cucchiara S, De Ridder L, et al. ESPGHAN revised porto criteria for the diagnosis of inflammatory bowel disease in children and adolescents. J Pediatr Gastroenterol Nutr. 2014; 58: 795-806.

19. Ducharme-Bénard S, Côté-Daigneault J, Lemoyne M, Orlicka K, Lahaie $\mathrm{R}$, Weber A, et al. Patients with inflammatory bowel disease are unaware of the impact of smoking on their disease. J Clin Gastroenterol. 2016; 50: 490-7.

20. De Bie C, Ballet V, Hendriks N, Coenen S, Weyts E, Van Assche G, et al. Smoking behaviour and knowledge of the health effects of smoking in patients with inflammatory bowel disease. Aliment Pharmacol Ther. 2015; 42: 1294-302.

21. Saadoune N, Peyrin-Bieroulet L, Baumann C, Bigard M.-A, Wirth N, Martinet Y. Beliefs and behaviour about smoking among inflammatory bowel disease patients. Eur J Gastroenterol Hepatol. 2015; 27: 797-803.

22. Ryan W. Patients with crohn's disease are unaware of the risks that smoking has on their disease. J Gastrointest Surg. 2003; 7: 706-11.

23. Pesce G, Marcon A, Calciano L, Perret JL, Abramson MJ, Bono R, et al. Time and age trends in smoking cessation in Europe. Manzoli L, editor. PLoS One 2019; 14: e0211976.

24. Wahed M, Goodhand JR, West O, McDermott A, Hajek P, Rampton DS. Tobacco dependence and awareness of health risks of smoking in patients with inflammatory bowel disease. Eur J Gastroenterol Hepatol. 2011; 23: 90-4.

25. (WHO) WHO. The current status of the tobacco epidemic in Poland. Copenhagen WHO Reg Off Eur. 2009.

26. Haug S, Schaub MP, Schmid H. Predictors of adolescent smoking cessation and smoking reduction. Patient Educ Couns. 2014; 95: 378-83.

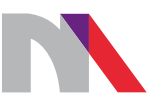

\section{Ministry of Science and Higher Education}

Republic of Poland

Generation of the DOI (Digital Object Identifier) - task financed under the agreement No. 618/P-DUN/2019 by the Minister of Science and Higher Education 